\title{
Tropylium Salt Promoted Hydroboration Reactions: Mechanistic Insights via Experimental and Computational Studies
}

\author{
Nhan Nu Hong Ton, ${ }^{[a]}$ Binh Khanh Mai ${ }^{*[b]}$ and Thanh Vinh Nguyen ${ }^{*[a]}$
}

\author{
[a] N. N. H. Ton, Dr T. V. Nguyen \\ School of Chemistry \\ University of New South Wales Sydney, NSW 2052, Australia \\ E-mail: t.v.nguyen@unsw.edu.au \\ [b] Dr B. K. Mai \\ Department of Chemistry \\ University of Pittsburgh, Pittsburgh, PA 15260, United States \\ Email: binh.mai@pitt.edu
}

Supporting information for this article is given via a link at the end of the document.

Abstract: Hydroboration reaction of alkynes is one of the most
synthetically powerful tools to access organoboron compounds,
versatile precursors for cross coupling chemistry. This type of reaction
has traditionally been mediated by transition metal or main group
catalysts. Herein, we report a novel method using tropylium salts,
typically known as organic oxidants and Lewis acids, to efficiently
promote the hydroboration reaction of alkynes. A broad range of
vinylboranes can be easily accessed via this metal-free protocol.
Similar hydroboration reactions of alkenes and epoxides can also be
efficiently catalyzed by the same tropylium catalysts. Experimental
studies and DFT calculations suggested that the reaction follows an
uncommon mechanistic paradigm, which is triggered by a hydride
abstraction of pinacolborane with tropylium ion. This is followed by a
series of in situ counterion-activated substituent exchanges to
generate boron intermediates that promote the hydroboration reaction

\section{Introduction}

Hydroboration of C-C multiple bonds is one of the most frequently used reactions in organic synthesis. ${ }^{[1]}$ It produces alkyl or alkenyl boranes, which are stable but versatile synthetic precursors for a wide range of coupling reactions. ${ }^{[1]}$ Since the early examples of uncatalyzed addition of borane to olefins by Brown, ${ }^{[2]}$ the field has evolved significantly over the last few decades to develop more efficient and practical catalytic systems for more challenging hydroborative processes. Among them, transition metal catalyzed hydroboration reaction remains the most thoroughly explored direction $^{[3]}$ (Scheme 1a) with the focus slowly shifts from precious metals ${ }^{[4]}$ to more abundant ones. ${ }^{[5]}$ More recently, main group catalytic hydroboration has emerged as a new research field,,$^{[6]}$ with organoborane catalysts being the most attractive options (Scheme 1b). ${ }^{[7]}$ The quest to make hydroboration reaction completely metal-free ventures further into some scattered examples of organocatalytic systems such as carboxylic acids ${ }^{[8]}$ or amides. ${ }^{[9]}$ While the mechanism of transition metal and main group element catalyzed hydroboration reactions has been extensively studied, there is a limited understanding of activation mode and reaction design in organocatalytic systems. Therefore, a reliable and efficient organocatalyst, which is also robust and

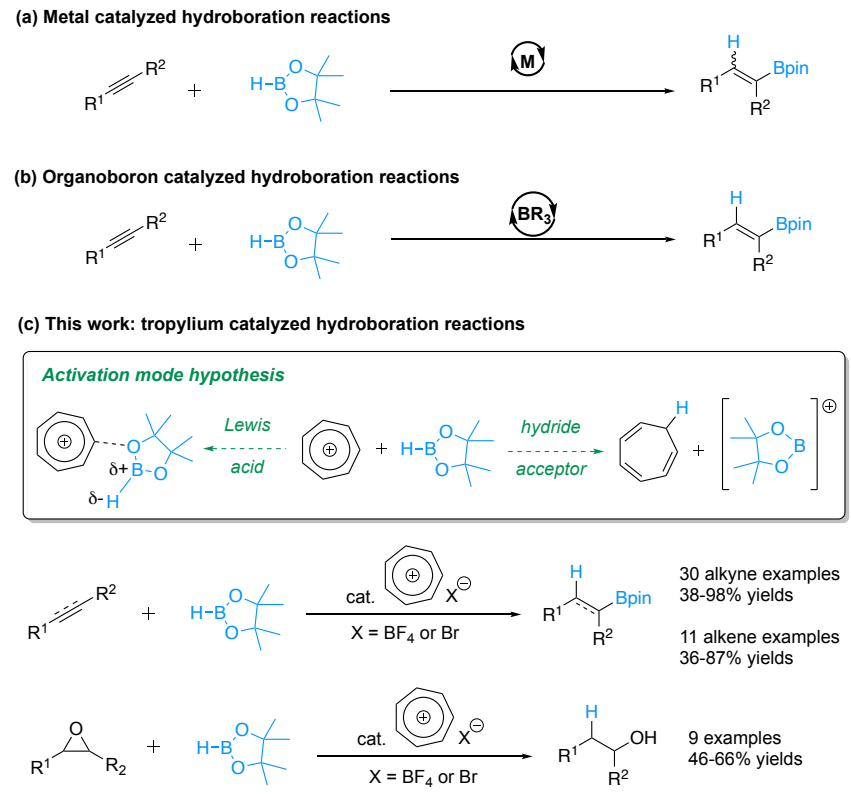

Scheme 1. Tropylium catalyzed hydroboration of alkynes, alkenes and epoxides.

simple for necessary mechanistic studies, is in demand.

Given our ongoing interest in the chemistry of tropylium ion ${ }^{[10]}$ and our recent recognition of the similarity in Lewis acid catalyst activity of tropylium ion ${ }^{[11]}$ to boron Lewis acids $B\left(\operatorname{Ar}^{F}\right)_{3}$, which are known as efficient catalysts for hydroboration reaction, ${ }^{[7,, 7 e-g, 7 i, 7 k]}$ we envisioned that tropylium salts could potentially serve as suitable organocatalysts for the same chemical transformation. In addition to its Lewis acidity, tropylium ion is also known as an organic oxidant, ${ }^{[12]}$ which could abstract a hydride from borane reagents to trigger the reaction via the formation of borenium cation (Scheme 1c). If this works, it will offer a new catalytic paradigm in hydroborative chemistry, especially when the electrophilic tropylium ion is distinctively different from existing catalysts, many of which are nucleophiles or hydride donors. 


\section{Optimization and Substrate Scope}

We started our investigation by looking at the catalytic activity of tropylium tetrafluoroborate (1a) in the reaction between phenylacetylene (1a) and pinacolborane (HBpin, 3). Gratifyingly, the initial reaction met with successful results. We proceeded further to optimize the reaction conditions on solvent, temperature stoichiometry of HBpin and tropylium catalyst loading (for full optimization details, see the ESI). ${ }^{[13]}$ The optimal conditions were presented in Table 1, where best reaction outcomes were achieved with $2 \mathrm{~mol} \% 1 \mathrm{a}$ at $70{ }^{\circ} \mathrm{C}$ in neat condition. The use of relatively non-polar solvents such as 1,2-dichloroethane or toluene also led to excellent yields of hydroboration adduct $\mathbf{4 a}$, while acetonitrile or THF completely turned off the reaction (entries 6-7, Table 1). Our initial thought is that this can be attributed to the fact that these solvents can solvate or coordinate very well to tropylium or boron intermediates, rendering them much less reactive for the reaction to proceed.

Without the tropylium catalyst, it only led to $15 \%$ yield of $4 a$ as the background reaction (entry 1). Tropylium hexafluorophosphate seemed to be much less effective for this reaction (entry 8$)$. Other tropylium salts such as chloride, bromide (1b) or triflate (1c) also proved to be capable of catalyzing the reaction with similar efficiency, however, tropylium chloride is too hygroscopic to be considered a practical catalyst. The important role of tropylium ion was highlighted by the fact that potassium or tetrabutylammonium salts of the same counterions either showed no catalytic activity or hindered the reaction. Furthermore, other hydride abstractors or organic Lewis acids such as tritylium ${ }^{[14]}$ or nitrosonium ${ }^{[15]}$ salts could also catalyze the reaction but with lower efficiencies than tropylium salts (entry 8).

The regioselectivity and stereochemistry of the reaction product was confirmed by comparison to literature data and also by a simple isotope labeling study. This study used our developed optimal reaction conditions on deuterated phenylacetylene (2a-D, $95 \%-D$, Scheme 2 ), which gave product $4 a-D$ in $87 \%$ yield with $93 \%$ deuterium at the terminal carbon, confirming that the HBpin added to the $\mathrm{C}-\mathrm{C}$ triple bond in cis-fashion with the indicated regioselectivity. We subsequently applied the optimized conditions towards the hydroboration of other alkyne substrates
Table 1. Optimization of tropylium-catalyzed reaction between $\mathbf{2 a}$ and $\mathbf{3}^{[\mathrm{a}]}$

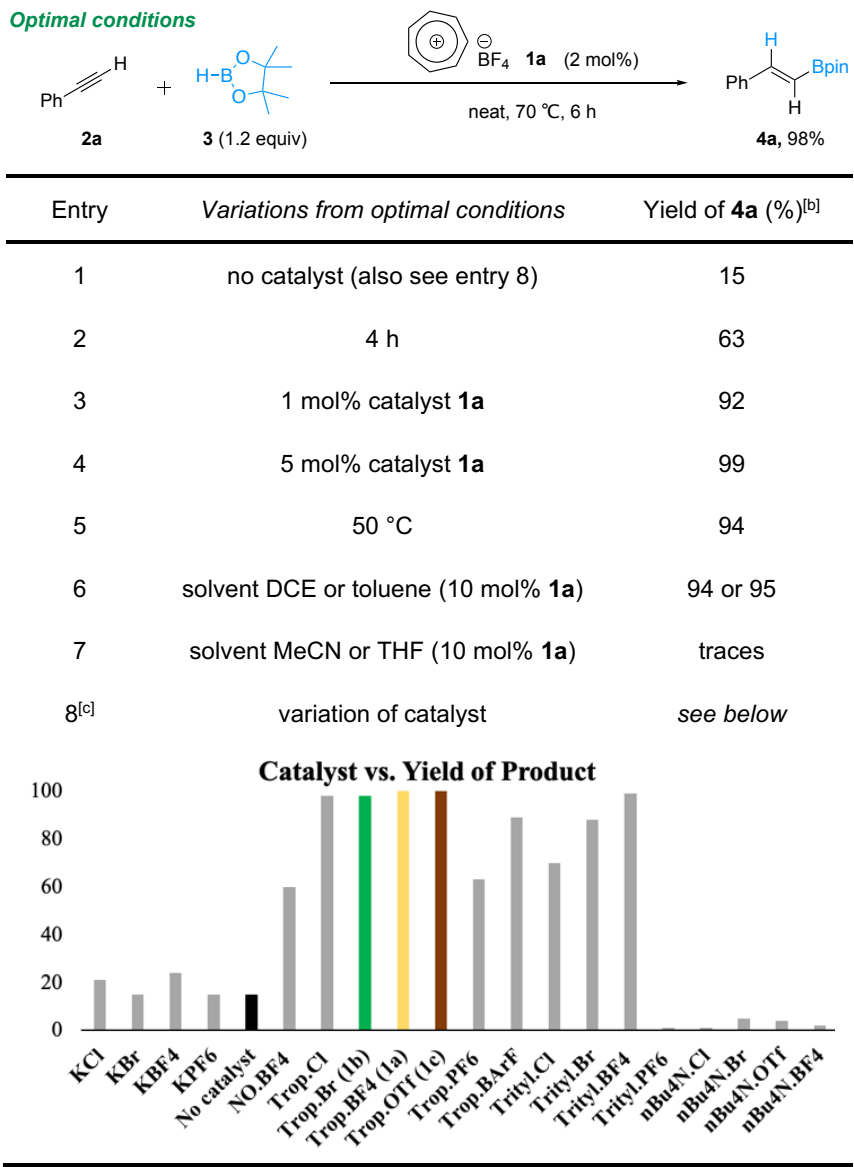

[a] Reaction conditions: $0.5 \mathrm{mmol} 2 \mathrm{a}, 0.6 \mathrm{mmol} 3$ (1.2 equiv) and $0.01 \mathrm{mmol} \mathbf{1 a}$ neat or in the indicated solvent $(2 \mathrm{~mL})$ at the indicated temperature for the indicated time. ${ }^{[13]}[\mathrm{b}]$ Yield of the isolated 4a. [c] All catalysts are anhydrous.

(Scheme 2). While these conditions work well for most substrates we tested, some of them showed unsatisfactory conversions. Therefore, we settled with a more suitable set of conditions from entry 4, Table 1 with $5 \mathrm{~mol} \%$ tropylium catalyst and 12 hours reaction time to ensure good efficiency is achieved across the

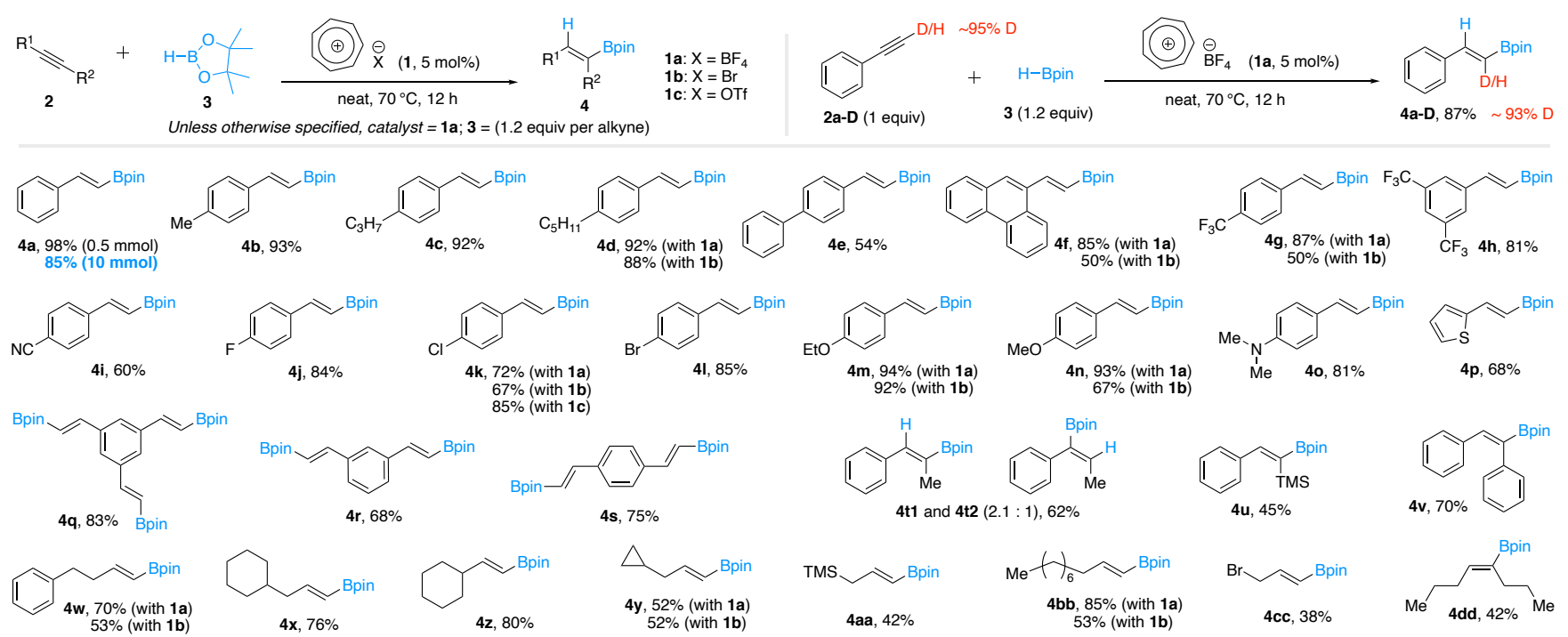

Scheme 2. Substrate scope of the hydroboration of alkynes. All products are trans-isomers, based on comparison of ${ }^{1} \mathrm{H}$ NMR spectra with literature data. 
the whole substrate scope. Most of arylacetylene substrates reacted smoothly to give the $(E)$-alkenyl boronic ester products in good to excellent yield (4a-4o, Scheme 2). The reaction worked efficiently with heterocyclic as well as bis and tris(acetylenyl) substrates (4p-4s). Non-terminal aromatic alkynes also reacted but led to lower product yields (4t-4v) and mixture of regioisomers (4t1 and 4t2). Under these reaction conditions, alkyl-substituted alkynes resulted in generally lower conversions to the products than their aryl alkyne counterparts.

\section{Mechanistic studies}

Apart from demonstration of practicality, it is also important to elucidate the mechanistic pathway of our newly developed tropylium salt catalyzed hydroboration of alkynes. While we carried out this project, the Thomas group reported important recognitions of hidden pathways in hydroboration chemistry, ${ }^{[16]}$ which actually involve the catalytic activity of in situ generated $\mathrm{BH}_{3}$ from nucleophilic catalysts or ligands and HBpin. These reports prompted us to carefully reconsider our original hypotheses and perform an in-depth investigation into the activation mode of our tropylium catalysts (Scheme 3). Thomas and co-workers nominated a qualitative 'hidden $\mathrm{BH}_{3}$ catalysis' test, involving the addition of tetramethylethylenediamine (TMEDA) to the reaction. This additive will supposedly complex with any $\mathrm{BH}_{3}$ formed in the reaction mixture, making it easier to identify by characteristic signals in NMR spectra. Thus, this was the first mechanistic control study that we did and we learned straightaway that it turned off the reaction completely, as no hydroboration product was formed in the reaction (Scheme 3a). In hindsight, this was not surprising, as tropylium ion is known to coordinate to the nitrogen centre of the amino group and even remove hydride from tertiary amines. ${ }^{[10 e, 12 a, 12 b]}$ The coordination of tropylium to TMEDA was observed by NMR spectroscopy (see page S39 in the experimental ESI); this presumably rendered our catalytic system inactive. Therefore, Thomas's qualitative test is, unfortunately, not applicable to our reaction conditions in this work. (a) Qualitative hidden $\mathrm{BH}_{3}$-catalysis tes

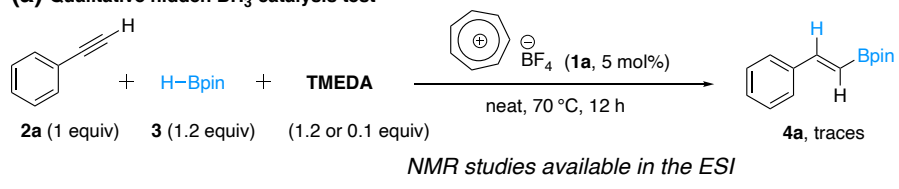

(b) $1^{\text {st }}$ mechanistic hypothesis

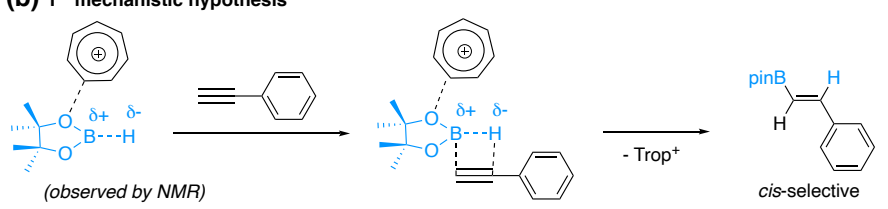

(c) $2^{\text {nd }}$ mechanistic hypothesis

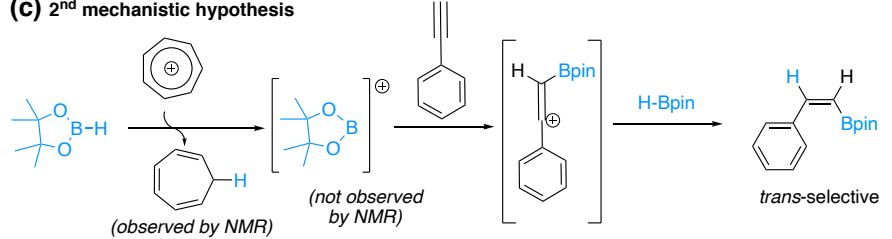

(d) ${ }^{1} \mathrm{H}$ NMR (400 MHz, $\left.\mathrm{CD}_{3} \mathrm{CN}, 298 \mathrm{~K}\right): 1 \mathrm{a}+\mathrm{HBpin}(1: 1)$ at rt after $30 \mathrm{~min}$

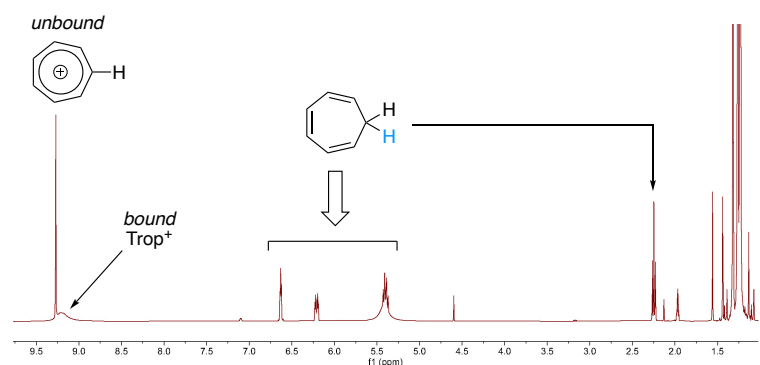

(e) ${ }^{11} \mathrm{~B} \mathrm{NMR}\left(128 \mathrm{MHz}, \mathrm{CD}_{3} \mathrm{CN}, 298 \mathrm{~K}\right): 1 \mathrm{a}+\mathrm{HB}$ in $(1: 4)$ at rt after $30 \mathrm{~min}$

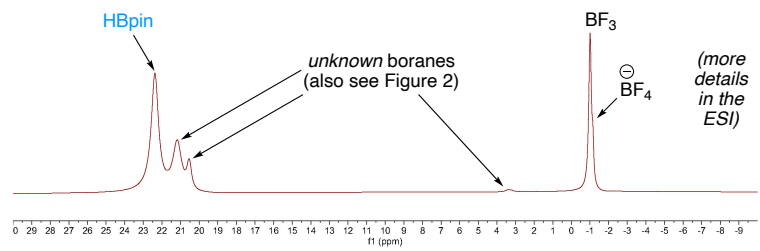

Scheme 3. Experimental mechanistic studies and original hypotheses of reaction mechanism: (a) Control study with TMEDA; (b) Lewis acid activation hypothesis; (c) Hydride abstraction activation hypothesis; (d) ${ }^{1} \mathrm{H}$ NMR complexation studies and (e) ${ }^{11} \mathrm{~B}$ NMR complexation studies between $\mathrm{HBpin}$ and $1 \mathrm{a}$.

Our first original hypothesis was that the Lewis-acidic tropylium ion could coordinate to the oxygen centres on HBpin, enhancing the polarization of the $\mathrm{B}-\mathrm{H}$ bond and promote the addition to the triple bond (Scheme $3 \mathrm{~b}$ ). This will likely produce the cis-selective addition product (trans-isomer), which agrees with our actual results. Our complexation NMR studies between tropylium salts and HBpin did show some supporting evidence for this Lewis coordination (Scheme 3d). However, there is a more predominant transformation happening between these two species. The ${ }^{1} \mathrm{H}$ NMR spectrum (in $\mathrm{CD}_{3} \mathrm{CN}$ ) of a $1: 4$ mixture of $\mathrm{Trop}^{\mathrm{B}} \mathrm{BF}_{4}$ 1a or Trop. $\mathrm{Br} 1 \mathrm{~b}$ with $\mathrm{HBpin}$ (to mimic $5 \mathrm{~mol} \%$ catalyst vs. 0.2 equiv $=$ $20 \mathrm{~mol} \%$ in excess of HBpin in optimal reaction conditions) also showed clear evidence of the formation of cycloheptriene, even when the analysis was done instantly after mixing the two compounds. Similar outcomes were observed with longer reaction times or at $70{ }^{\circ} \mathrm{C}$ (see Scheme $3 \mathrm{~d}$ for a representative spectrum of the mixture after 30 minutes at room temperature). It beckoned another hypothesis we had at the beginning of this work that tropylium ion could act as a hydride abstractor ${ }^{[12 a, 12 b]}$ to generate a borenium ion from HBpin. ${ }^{[4 e, 17]}$ This could then quickly react with the alkyne to form a vinyl cation intermediate, ${ }^{[17 \mathrm{~b}, 18]}$ which subsequently take a hydride from another molecule of HBpin to generate the target product and start another reaction cycle (Scheme 3c). ${ }^{[7 d]}$ It should be noted that this reaction pathway would tentatively produce the trans-selective hydroboration product (cis-isomer), which contradicts our experimental outcomes.

However, the ${ }^{11} \mathrm{~B}$ NMR of the Trop. BF 4 1a/HBpin mixture (Scheme $3 e$ ) did not show the presence of a borenium species. This is most likely due to the fact that the borenium ion would immediately combine with a nucleophilic species in the reaction mixture, such as the fluoride from tetrafluoroborate or the bromide, to form more stable boranes. Apart from remaining $\mathrm{HBpin}_{\text {and }} \mathrm{BF}_{4}$ anion, we initially could only identify the presence of $\mathrm{BF}_{3}$, plus the 
formation of some unidentified boranes in the ${ }^{11} \mathrm{~B}$ NMR spectrum (Scheme $3 \mathrm{e}$, also see Figure 2 and discussion later). On the other hand, we have carried out an extensive Density Functional Theory (DFT) calculation ${ }^{[19]}$ to validate our hypotheses in either Scheme $3 \mathrm{~b}$ or 3c. Our DFT calculations showed that the activation barrier for the uncatalyzed hydroboration between HBpin and alkyne 2a is approximately $42.5 \mathrm{kcal} / \mathrm{mol}$, which is consistent with the fact that this hydroboration cannot occur without catalyst (see Figure S1 in the computational ESI for details). However, all transition states and intermediates related to mechanistic hypotheses in Schemes $3 b$ and $3 c$ are too energetically unfavored to be feasible (see Figure $\mathrm{S} 1$ in the computational ESI); therefore, they could be considered unlikely for the reaction.

In our substrate scope study discussed above in Scheme 2, tropylium bromide (1b) and triflate (1c) catalysts were also employed for a selected number of substrates and gave comparable or slightly lower efficiencies than tropylium tetrafluoroborate. It should be noted that we chose not to carry out more examples with these catalysts due to their hygroscopic and difficult-to-handle nature. On the other hand, as discussed earlier in Table 1, potassium or tetrabutylammonium salts of the same counterions either showed no catalytic activity or hindered the reaction while tritylium or nitrosonium salts could also catalyze the reaction but with lower efficiencies than tropylium salts. It prompted us to investigate the possibility of a more direct role of tropylium ion in triggering the reaction, which is depicted in Scheme 4 . We hypothesized that the electrophilic tropylium ion could add to the $\mathrm{C}-\mathrm{C}$ triple bond to generate a vinyl cation intermediate 5 , which can take a hydride from HBpin similar to the borenium pathway in Scheme 3c. This would result in closely associated pair 6 , which would lead to the formation of carbocation 7 . Intermediate 7 could then eliminate a tropylium ion to form the hydroboration product.

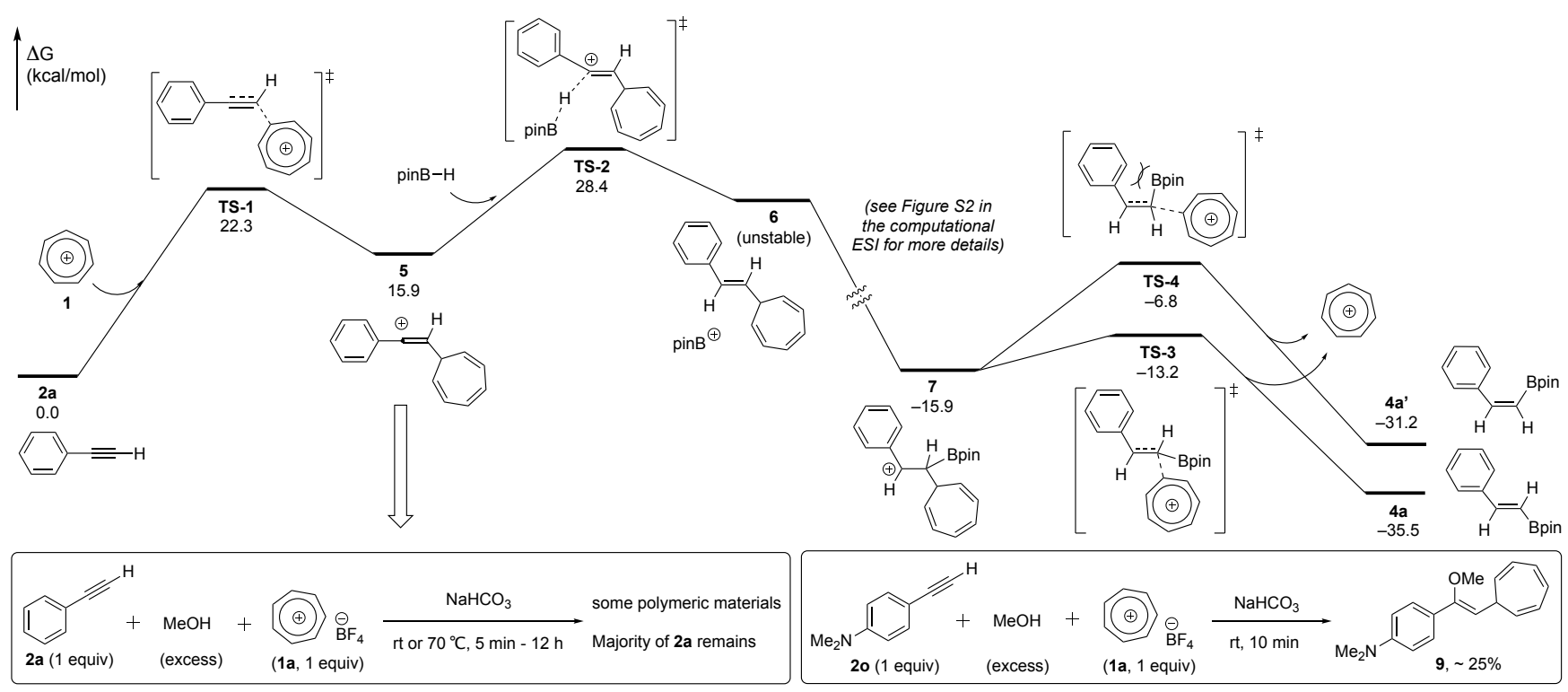

Scheme 4. The third mechanistic hypothesis and supporting computational and experimental studies.

A combination of computational and experimental studies was then carried out to find supporting arguments for this new hypothesis (Scheme 4). The addition of tropylium ion to the C-C triple bond of 2a has an activation energy of $22.3 \mathrm{kcal} / \mathrm{mol}$ and that of the subsequent hydride abstraction step between vinyl cation 5 and HBpin is calculated to be $28.4 \mathrm{kcal} / \mathrm{mol}$ (Scheme 4). The hydride transfer presumably leads to the formation of the associated pair $\mathbf{6}$. Our attempt to optimize 6 always generate carbocation 7 , which suggests that 6 is unstable and will transform into 7 barrierlessly. To confirm this result, Born-Oppenheimer molecular dynamics (BOMD) simulations were carried out. We found that after TS-2, 6 is formed and rapidly converted to 7 without crossing any barrier at around 248 fs (see Figure S2 in the computational ESI), which is consistent with our DFT calculations on this species.

While TS-2 is associated with high activation energy, making this a challenging pathway, the rest of this reaction profile looks rather feasible. Because of the steric repulsion between Bpin and phenyl moieties, TS-3 is calculated to be $6.4 \mathrm{kcal} / \mathrm{mol}$ lower in energy than that of TS-4, resulting in the cis-selective $4 a$ as the favored product, which is consistent with our experiments. However, we failed to find experimental evidence of the formation of vinyl cation 5 by trapping it with other nucleophiles. As a representative example, we could not find the methoxy-cycloheptatrienylation adduct when we replaced the borane reagent with methanol (Scheme 4, bottom-left). Interestingly, a similar trapping reaction with the electron-rich aromatic alkyne 20 successfully led to the formation of adduct $\mathbf{9}$ in decent efficiency without optimization of reaction conditions (Scheme 4, bottom-right). The formation of 9 indicates that when there is a strong stabilization effect, in this case coming from the conjugation of $p-\mathrm{NMe}_{2}$ group, it is possible to form a vinyl cation intermediate (50), which reacts with methanol to form $\mathbf{9}$. However, this type of reactivity might not be general for the other alkyne substrates, as observed with the standard substrate $\mathbf{2 a}$. In addition to the high activation energy to TS-2, although this does not rule out completely but casts some doubts over our third hypothesis of this tropylium-promote hydroboration reaction mechanism. 
Another revisit to our catalyst scope in Table 1 revealed that tropylium salts with chloride, bromide, triflate and tetrafluoroborate counterions catalyzed the reaction efficiently, while those with hexfluorophosphate or BArF counterions gave unsatisfactory results. Furthermore, while tritylium tetrafluoroborate led to almost the same efficiency as the tropylium counterpart, tritylium hexafluorophosphate completely suppressed this hydroboration reaction. These results indicated that while tropylium ion is an essential component, the counterions have an undeniable role in their catalytic activity. So to further unravel the mechanism of our chemistry, we carried out kinetic studies of reactions catalyzed by $5 \mathrm{~mol} \%$ of tropylium bromide (1b) and different loadings of tropylium tetrafluoroborate (1a) under optimal conditions. The conversion to the hydroboration product was monitored by ${ }^{1} \mathrm{H}$ NMR spectroscopy over time (Figure 1a). The interesting thing we learned from these studies is that most reactions (except the one with high catalyst loading $-15 \mathrm{~mol} \%$ of $1 \mathrm{a}$ ) seemed to undergo a catalyst preactivation period of 5-10 minutes. The lower catalyst loading was, the longer the reaction took to start showing some conversion. The pre-activation time of $5 \mathrm{~mol} \%$ tropylium bromide was similar to that of $10 \mathrm{~mol} \%$ tropylium tetrafluoroborate. This prompted us to look deeper into the reaction between tropylium salts and HBpin, which was presumably the only chemical process that caused this pre-activation period.

As discussed before, it was obviously evident that tropylium ion abstracted a hydride from HBpin and turned into cycloheptatriene, but what became of the rest eluded us earlier. The pinacolatoborenium ion intermediate (see Scheme 3c) was never observed so we believed it was captured by some nucleophiles in the reaction mixture. This is presumably where the counterions from tropylium salts came into play. With tropylium tetrafluoroborate, it was previously reported that the $\mathrm{BF}_{4}$ anion can dissociate to form $\mathrm{BF}_{3}$ and fluoride, which can capture the borenium ion into the corresponding fluoropinacolato borane. ${ }^{[10 f]}$ Similar reaction can presumably happen with tropylium bromide to form bromopinacolato borane.

Therefore, we decided to carry out further ${ }^{11} \mathrm{~B}$ and ${ }^{19} \mathrm{~F}$ NMR spectroscopic analyses of the reaction between tropylium salts and HBpin to get more insights. The pertinent sections of ${ }^{11} \mathrm{~B}$ NMR spectra of HBpin and tropylium tetrafluoroborate (1a) mixture at

\section{(a) Conversion to $4 a$ (\%) vs. Reaction time (min)}

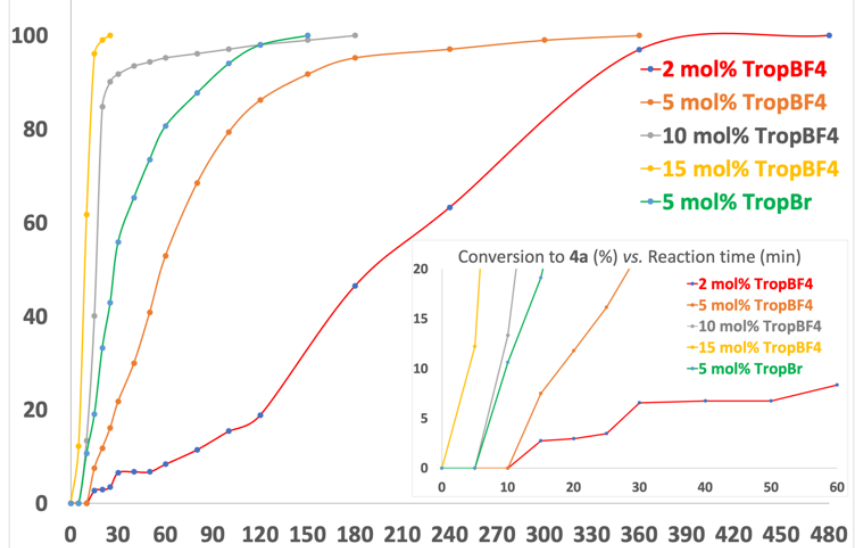

Figure 1. (a) Kinetic studies of the conversion of $\mathbf{2} \mathbf{a}$ to reaction product $\mathbf{4 a}$ with different catalyst loadings, (inset) zoom-in version of initial reaction stage. (a) ${ }^{11} \mathrm{~B}$ NMR $\left(128 \mathrm{MHz}, 298 \mathrm{~K}, \mathrm{CD}_{3} \mathrm{CN}\right)$

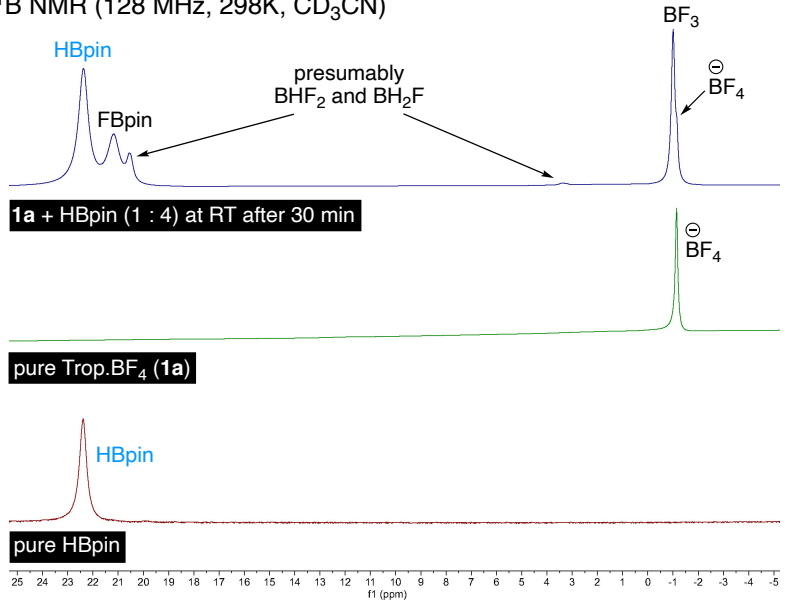

(b) ${ }^{19} \mathrm{~F} \mathrm{NMR}\left(376.5 \mathrm{MHz}, 298 \mathrm{~K}, \mathrm{CD}_{3} \mathrm{CN}\right)$

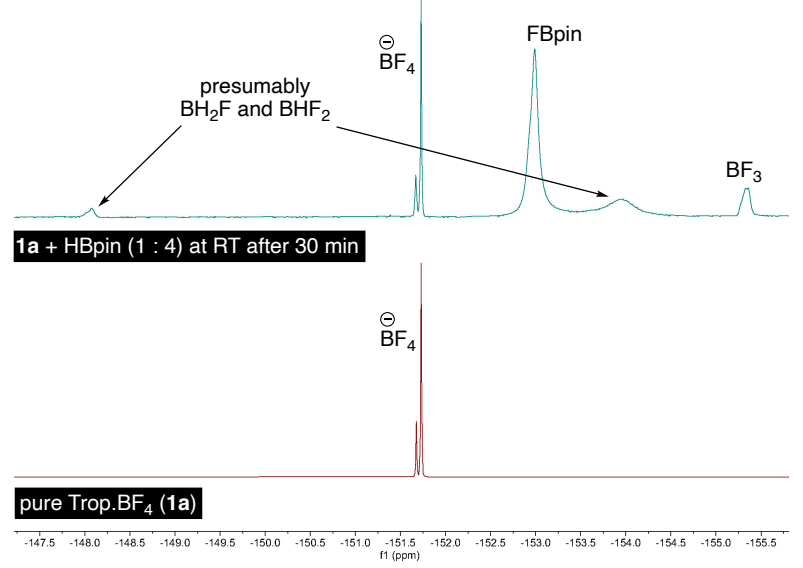

Figure 2. ${ }^{11} \mathrm{~B}\left(128 \mathrm{MHz}, \mathrm{CD}_{3} \mathrm{CN}, 298 \mathrm{~K}\right)$ and ${ }^{19} \mathrm{~F}\left(376.5 \mathrm{MHz}, \mathrm{CD}_{3} \mathrm{CN}, 298 \mathrm{~K}\right)$ NMR spectra of HBpin and $1 \mathrm{a}$ (pure and mixture at $4: 1$ ratio, rt, $30 \mathrm{~min}$ ).

$4: 1$ ratio after 30 minutes at room temperature can be seen in Figure 2. ${ }^{[20]}$ After matching signals with known boron species in literature, we were able to identify FBpin ${ }^{[21]}\left(\sim 21 \mathrm{ppm}\right.$ in ${ }^{11} \mathrm{~B}$ and $\sim-153 \mathrm{ppm}$ in ${ }^{19} \mathrm{~F}$ NMR) at all ratios. We did not observed any signals corresponding to [FHBpin]-, formed by the coordination of fluoride to HBpin, which was reported by the Thomas group in their work with iron and cobalt tetrafluoroborate salts. ${ }^{[22]}$ There were signals corresponding to some remaining $\mathrm{BF}_{4}$ anion $(\sim-1$ ppm and $\sim-151.7 \mathrm{ppm})$ and the by-product $\mathrm{BF}_{3}(\sim-1$ and $\sim-$ $155.3 \mathrm{ppm})$. There were two more new boron compounds formed in this mixture. Although we could not fully interpret these signals, we believe that they correspond to $\mathrm{BHF}_{2}{ }^{[23]}$ and $\mathrm{BH}_{2} \mathrm{~F}$ (never been characterized by experimental NMR spectroscopy before), ${ }^{[24]}$ which were presumably produced via substituent exchange reactions between $\mathrm{HB} p i n$ and $\mathrm{BF}_{3}$ (vide infra). ${ }^{[7 e, 25]}$ It should be noted that in ${ }^{19} \mathrm{~F}$ NMR spectra, there are signals corresponding to ${ }^{10} \mathrm{~B}$ (small peaks or shoulders) and ${ }^{11} \mathrm{~B}$ (major peaks) isotopes for each species (see page S41 in the experimental ESI).

With our experimental data pointing towards the formation of a complex mixture of a range of borane species in the presence of HBpin and tropylium tetrafluoroborate, we carried out computational studies to rationalize these results. Interestingly, our DFT calculations ${ }^{[19]}$ show that HBpin can indeed react with tropylium tetrafluoroborate via hydride transfer transition state TS- 
5 to form cycloheptriene, FBpin, and $\mathrm{BF}_{3}$ species with the calculated barrier of $25.3 \mathrm{kcal} / \mathrm{mol}$ (Figure 3a). This result is consistent with experimental finding that other hydride acceptors, including tritylium and nitrosonium tetrafluoroborate, can also promote this hydroboration reaction albeit with lower efficiency; whereas, potassium and tetrabutylammonium salts show no activity (Table 1). We also found that $\mathrm{BF}_{3}$ species can further react with $\mathrm{HBpin}$ via two transborylation transition states TS-6 and TS7 (B-H/B-F $\sigma$-bond metathesis), giving $\mathrm{BHF}_{2}$ and $\mathrm{BH}_{2} \mathrm{~F}$ species with the activation barriers calculated to be $27.6 \mathrm{kcal} / \mathrm{mol}$ relative to $\mathrm{BF}_{3}$ intermediate (Figure $3 \mathrm{a}$ ).

Our DFT calculations demonstrate that for this hydroboration of alkyne, the formation of in situ $\mathrm{BH}_{2} \mathrm{~F}$ species is very important and $\mathrm{BH}_{2} \mathrm{~F}$ can potentially act as a hidden boron catalyst. The catalytic cycle for the hydroboration is shown in Figure $3 \mathrm{~b}$. From $\mathrm{BH}_{2} \mathrm{~F}$, the 1,2-syn-addition to $2 \mathrm{a}$ via TS-8 is found to occur giving boron alkene intermediate 10 with the calculated barrier of $9.9 \mathrm{kcal} / \mathrm{mol}$ relative to $\mathrm{BH}_{2} \mathrm{~F}$. To proceed, transborylation ( $\mathrm{B}-\mathrm{C} / \mathrm{B}-\mathrm{H} \sigma$-bond metathesis) between 10 and HBpin can then take place in a stepwise fashion via TS-9 and TS-10 giving product 4 a with the retention of $E$-configuration. The activation barriers of TS-9 and TS-10 are calculated to be 22.4 and $22.7 \mathrm{kcal} / \mathrm{mol}$ relative to intermediate 10. The transborylation involves a transient $\mu-\mathrm{H}$ bridged species 11 consisting of two three-center two-electron (" $3 \mathrm{c}-2 \mathrm{e}$ ") bonds, which are employed by the $s p^{2}$ hybrid orbital of bridging carbon atom and the $1 s$ orbital of bridging hydrogen atom $^{[7]]}$ (see Figure S3 in the computational ESI for the intrinsic bond orbital analysis of intermediate 11). Our DFT calculations are in agreement with a recent study by Thomas and Lloyd-Jones for the Arase-Hoshi alkyne hydoboration. ${ }^{[7]]}$

The activation barrier for the formation of $\mathrm{BH}_{2} \mathrm{~F}$ catalyst from HBpin and tropylium tetrafluoroborate is calculated to be 27.6 $\mathrm{kcal} / \mathrm{mol}$ (Figure 3a); whereas, the rate-determining step for the hydroboration of alkyne by $\mathrm{BH}_{2} \mathrm{~F}$ catalyst is $22.7 \mathrm{kcal} / \mathrm{mol}$ (Figure $3 b)$. These results suggest that the reaction has to suspend until the formation of $\mathrm{BH}_{2} \mathrm{~F}$. Once $\mathrm{BH}_{2} \mathrm{~F}$ species is generated, the hydroboration of alkyne can take place efficiently. This finding is consistent with our experimental data that an "activation period" exists at the beginning of the reaction (Figure 1). Furthermore, solvents that can strongly bind to the boron intermediates in Figure $3 a$ are likely to hinder the reaction. For example, the binding energy of THF to $\mathrm{BF}_{3}$ is calculated to be $12.9 \mathrm{kcal} / \mathrm{mol}$, which thus increases the activation barrier of TS-6 in THF to be $40.5 \mathrm{kcal} / \mathrm{mol}$ relative to $\mathrm{BF}_{3}-\mathrm{THF}$ complex. This result gives a rationalization for the negative effect of solvents on this hydroboration reaction (Table 1).

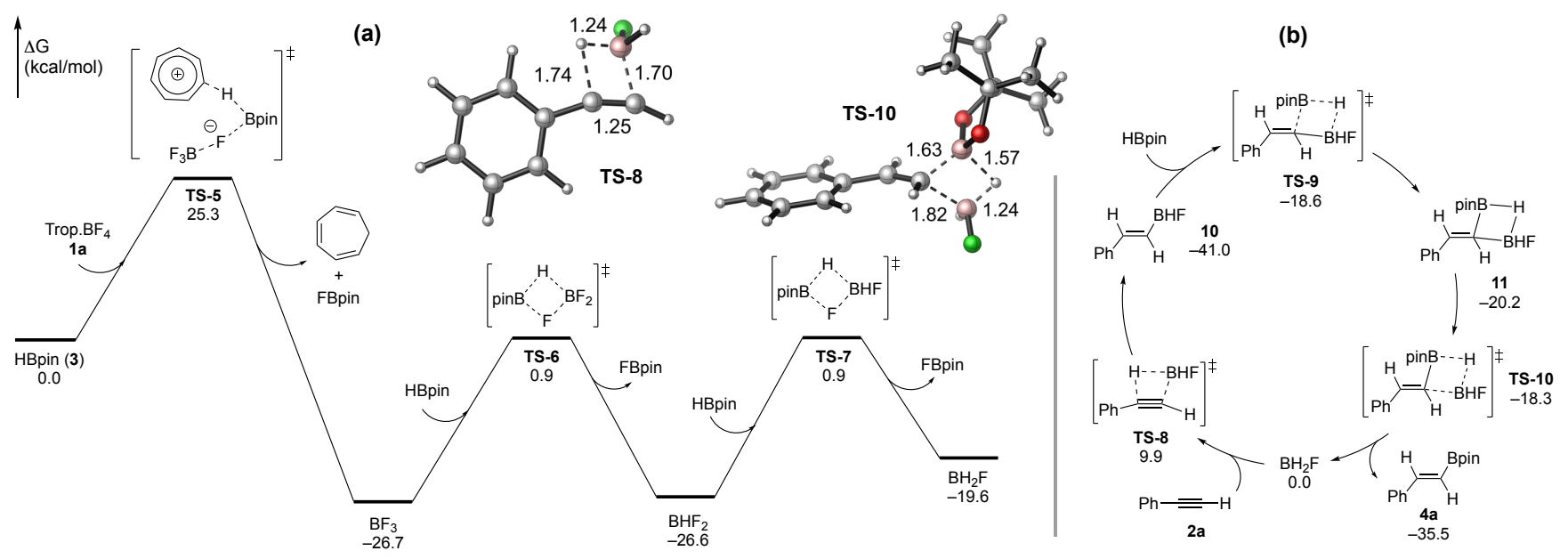

Figure 3. (a) Free energy profile ( $\mathrm{kcal} / \mathrm{mol}$ ) for the formation of $\mathrm{BH}_{2} \mathrm{~F}$ from $\mathrm{HBpin}$ and tropylium tetrafluoroborate (b) catalytic cycle for the hydroboration of alkyne catalyzed by $\mathrm{BH}_{2} \mathrm{~F}$. In Figure $3 \mathrm{~b}$, energy values $\left(\mathrm{kcal} / \mathrm{mol}\right.$ ) are relative to $\mathrm{BH}_{2} \mathrm{~F}$. All bond distances are in Ångstrom.

Here, it should be mentioned that we have also carried out DFT calculations for various hydroboration pathways that could be triggered by each boron intermediate of the reaction, including the possibilities that (i) $\mathrm{BHF}_{2}$ intermediate acts as the hydroboration catalyst and (ii) $\mathrm{BH}_{3}$ forms from further reduction of $\mathrm{BH}_{2} \mathrm{~F}$ by HBpin and subsequently acts as the catalyst. ${ }^{[16]}$ The catalytic cycle for the hydroboration of alkyne catalyzed by $\mathrm{BHF}_{2}$ is shown in Figure $\mathrm{S} 4$ in the computational ESI. The overall activation barrier for this catalytic cycle is calculated to be $30.4 \mathrm{kcal} / \mathrm{mol}$, which is indeed much less favorable than the same process with $\mathrm{BH}_{2} \mathrm{~F}$ (Figure 3b) and unlikely to happen. The reaction mechanism for the hydroboration catalyzed by $\mathrm{BH}_{3}$ is shown in Figure S5 in the computational ESI. It is obvious that $\mathrm{BH}_{3}$ can also efficiently catalyze the conversion of $\mathbf{2 a}$ to $\mathbf{4 a}$. However, because of the high reactivity of $\mathrm{BH}_{3}$ species, the reaction is unlikely to stop there. Intrinsic reaction coordinate (IRC) calculation and BOMD simulations indicate that at around $271 \mathrm{fs}$ after the transborylation giving $\mathrm{BH}_{3}$ and $\mathbf{4 a}, \mathrm{BH}_{3}$ species can quickly react to $\mathrm{C}-\mathrm{C}$ double bond of $4 \mathrm{a}$ without crossing any barrier giving an alkane intermediate (see Figure S6 and S7 in the computational ESI). This is followed by a transborylation with HBpin giving gemdiboron alkane as the favorable product. This is inconsistent with our experimental findings in that we can only observe the semihydroboration adduct $\mathbf{4 a}$ with our tropylium catalysts. A control studies using $\mathrm{BH}_{3}$ (commercially available in complexes with $\mathrm{Me}_{2} \mathrm{~S}$ or THF) as catalyst indeed led to the formation of gemdiboron alkane product. Moreover, the activation barrier for the transborylation between $\mathrm{BH}_{2} \mathrm{~F}$ and $\mathrm{HBpin}$ giving $\mathrm{BH}_{3}$ is calculated to be $8.1 \mathrm{kcal} / \mathrm{mol}$ higher than that of the 1,2-syn-addition between $\mathrm{BH}_{2} \mathrm{~F}$ and alkyne 2a (see Figure $\mathrm{S} 1$ in the computational ESI for detail), which indicates that $\mathrm{BH}_{3}$ is unlikely to be the catalyst for our hydroboration reaction. 
Our mechanism, therefore, can be considered a hybridization of the hidden catalysis by borane ${ }^{[16]}$ and the counterion-activated catalysis by $\mathrm{Fe} / \mathrm{Co}$ tetrafluorborate salts. ${ }^{[22]}$ Both of these were recently reported by the Thomas group as game-changing factors in hydroboration catalysis. While our mechanistic proposal still needs further validations in future work, the fact that a simple species such as tropylium tetrafluoroborate $\mathbf{1 a}$ could potentially trigger hydroboration in several different ways is intriguing.

As the hydroboration reaction can also work with tropylium bromide $\mathbf{1 b}$ as catalyst, it has to activate the system through a different pathway since there is no $\mathrm{BF}_{4}$ anion. Experiment finding suggests that $5 \mathrm{~mol} \%$ tropylium bromide can promote the hydroboration with similar kinetics and efficiency as $10 \mathrm{~mol} \%$ tropylium tetrafluoroborate (see Figure 1). Our DFT calculations suggest that HBpin can react with tropylium bromide via TS-11 to form BrBpin with the calculated barrier of $21.7 \mathrm{kcal} / \mathrm{mol}$ (Figure 4a). From BrBpin, two transborylation steps between BrBpin and two HBpin molecules then take place giving $\mathrm{BH}_{2} \mathrm{Br}$, and $\mathrm{B}_{2}$ pin 3 as the by-product, which will act as a catalyst for the hydroboration of alkyne (Figure 4a). The catalytic cycle for the hydroboration of alkyne $2 \mathrm{a}$ by $\mathrm{BH}_{2} \mathrm{Br}$ is shown in Figure $4 \mathrm{~b}$ and the reaction mechanism is found to be similar to the hydroboration with $\mathrm{BH}_{2} \mathrm{~F}$. The reaction starts with a 1,2-syn-addition via TS-14 between $\mathrm{BH}_{2} \mathrm{Br}$ and alkyne, which is followed by a stepwise transborylation via TS-15 and TS-16 giving alkenylboron product $4 a$. The hydroboration catalytic cycle by $\mathrm{BH}_{2} \mathrm{Br}$ in Figure $4 \mathrm{~b}$ has lower energy barriers than the one by $\mathrm{BH}_{2} \mathrm{~F}$ in Figure $3 \mathrm{~b}$, which is consistent with our experimental data that $\mathrm{Trop} . \mathrm{Br} \mathbf{1 b}$ led to faster reaction than the same catalyst loading of $\mathrm{Trop}_{\mathrm{BF}} \mathrm{BF}_{4}$ 1a (see Figure 1a). ${ }^{[26]}$
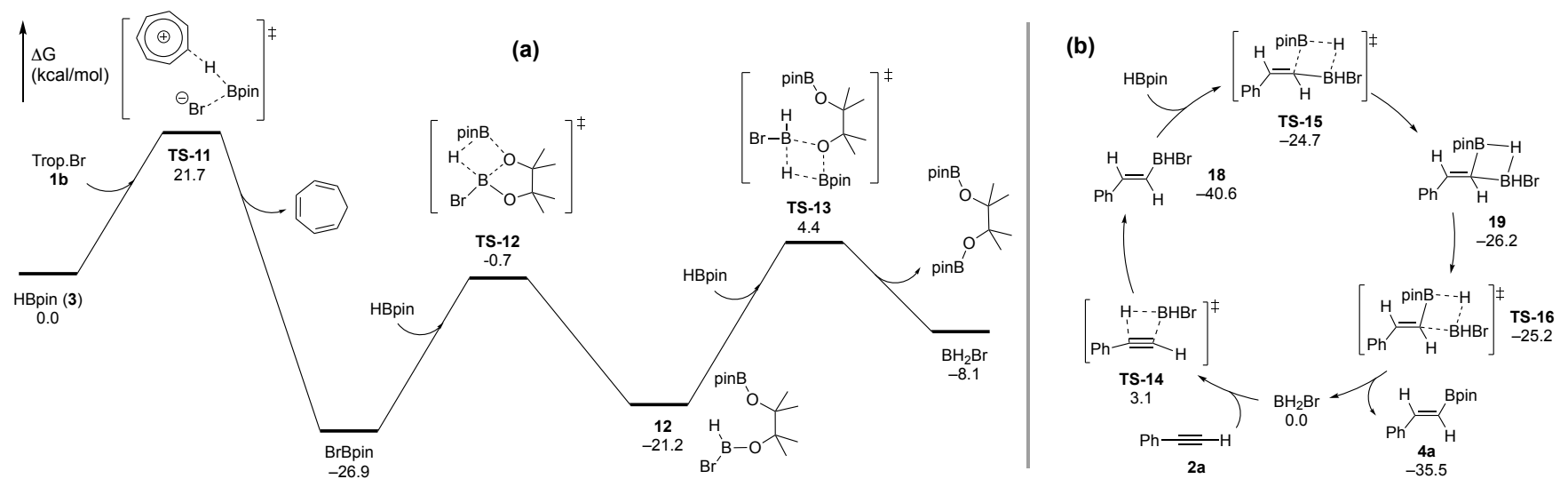

Figure 4. (a) Free energy profile (kcal/mol) for the formation of $\mathrm{BH}_{2} \mathrm{Br}$ from $\mathrm{HBpin}$ and tropylium bromide and (b) catalytic cycle for the hydroboration of alkyne catalyzed by $\mathrm{BH}_{2} \mathrm{~F}$ (energy values are relative to $\mathrm{BH}_{2} \mathrm{Br}$ ).

\section{Hydroboration of Alkenes and Epoxides}

Thus, a combination of experiments and DFT calculations suggest that HBpin can react with tropylium salts, i.e. tropylium tetrafluoroborate or bromide, to generate in situ boron catalysts, i.e. $\mathrm{BH}_{2} \mathrm{~F}$ and $\mathrm{BH}_{2} \mathrm{Br}$. We were curious to see if their catalytic activity can be amenable to other types of hydroboration reactions. We chose to investigate this on alkenes and epoxides, as they are more challenging substrates than carbonyl or imines compounds. $\left.{ }^{[1 \mathrm{~b},} 3 \mathrm{a}, 3 \mathrm{c}, 6 \mathrm{6l}\right] \mathrm{A}$ competitive reaction between phenylacetylene $\mathbf{2 a}$ and styrene $13 a$ with limiting amount of HBpin showed that the hydroboration of alkenes is less favorable than that of alkynes (Scheme 5a). However, with the same set of reaction conditions used in Scheme 2, we were able to hydroborylate a range of aromatic as well as aliphatic alkenes to the desired products with moderate to high yields. Tropylium salts $1 \mathrm{a}$ and $\mathbf{1 b}$ promoted the reaction with similar efficiencies on the substrates we selected for comparative studies (Scheme 5b).

We have also calculated the reaction mechanism for the hydroboration of alkene catalyzed by $\mathrm{BH}_{2} \mathrm{~F}$ species. This mechanism of this reaction is indeed similar to the hydroboration of alkyne where the first step is the 1,2-syn-addition to alkene 13, followed by a transborylation giving boron alkane product $\mathbf{1 4}$ (see Figure S8 in the computational ESI). Our calculations were also consistent with the fact that hydroboration of alkenes is more challenging than alkynes.

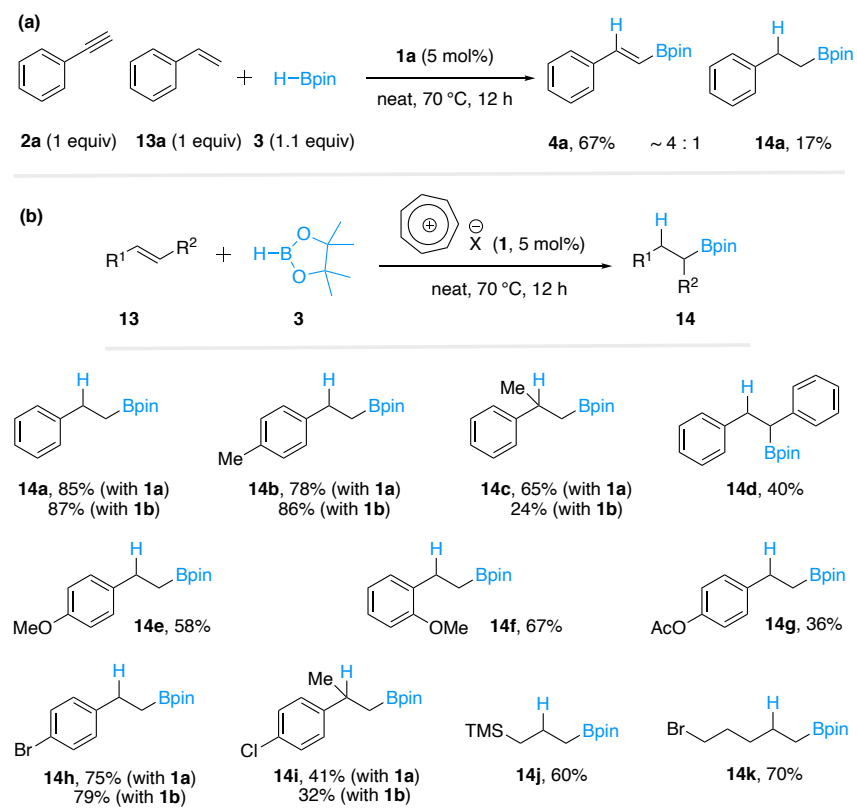

Scheme 5. (a) Competitive reactions between alkyne and alkene; (b) substrate scope of the hydroboration of alkenes. 
Similar to alkenes, tropylium-catalyzed hydroboration of epoxides was slightly more challenging than the reaction with alkynes. It required a higher catalyst loading of tropylium catalyst and longer reaction time to achieve complete conversion (Scheme 6a). It should be noted that the boronic ester products 16 were hydrolyzed to alcohols 17 during reaction workup for better purification process. ${ }^{[27]}$ However, due to this extra step, yields of the isolated alcohol products are not reflective of the reaction efficiency. Indeed, ${ }^{1} \mathrm{H}$ and ${ }^{11} \mathrm{~B}$ NMR analyses of the crude reaction mixtures before workup showed $90 \%$-quantitative conversion of the epoxides to their corresponding boronic esters.

We then focused on elucidating the reaction mechanism for the hydroboration of epoxides. The computed free energy profile is shown in Scheme 6b. It turns out that $\mathrm{BH}_{2} \mathrm{~F}$ can also act as a catalyst for this reaction. The reaction starts with a ring-opening transition state TS-17 giving zwitterionic species 21, followed by a hydride transfer from boron moiety to the carbocationic centre via TS-18 to form intermediate 22. To proceed, transborylation (B-O/B-H $\sigma$-bond metathesis) between 20 and HBpin in a concerted manner via TS-19 occurs to give product 16 . We could not locate any transient $\mu$-H bridged species in the transborylation step. The activation barriers of the hydride transfer TS-18 and the transborylation TS-19 are calculated to be 20.9 and $23.3 \mathrm{kcal} / \mathrm{mol}$ relative to 18 and 20 , respectively.

(a)

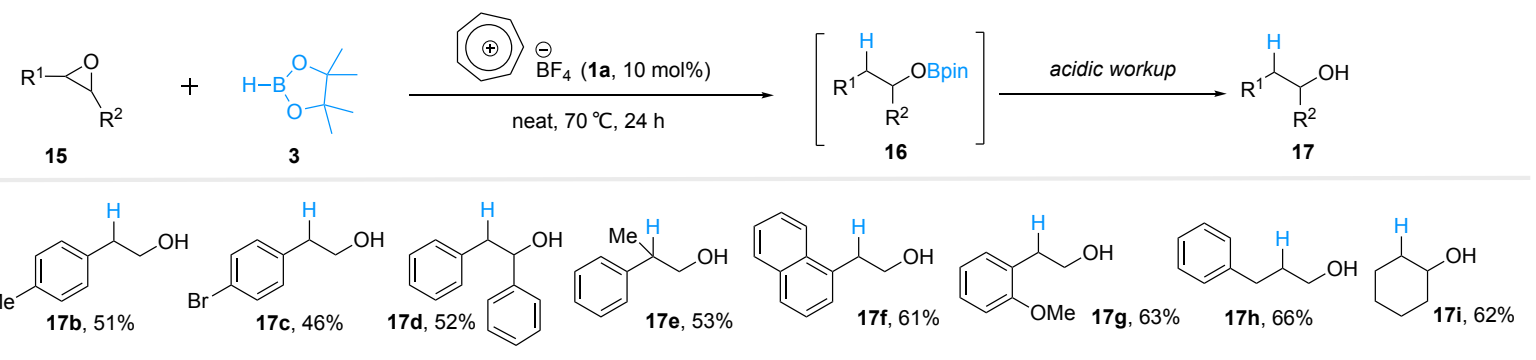

(b)

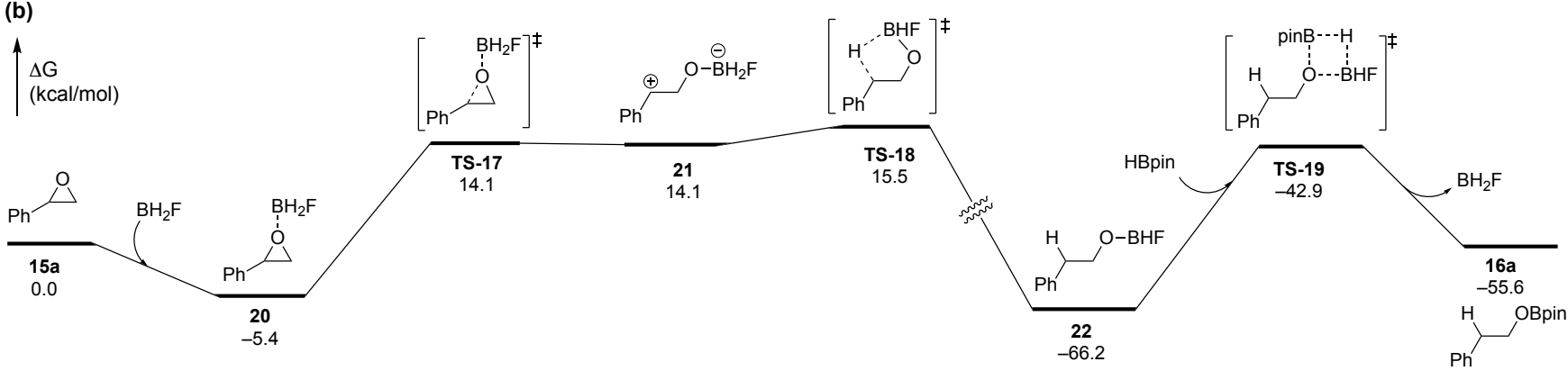

Scheme 6. (a) Substrate scope of epoxide hydroboration; (b) free energy profile ( $\mathrm{kcal} / \mathrm{mol})$ for the hydroboration of epoxide $15 \mathrm{catalyzed} \mathrm{by} \mathrm{BH}_{2} \mathrm{~F}$.

In conclusion, we report a novel hydroboration protocol using tropylium salts as efficient reaction promoters. This method is amenable to alkynes, alkenes and epoxides to produce a broad range of vinyl- and alkylboranes as well as alcohols. Apart from the synthetic value of this metal-free protocol, tropylium salts proved to be attractive and versatile probes to explore mechanistic details of hydroboration reaction. This work offers insights into the controversial topic of hidden or true catalysis for hydroboration reactions. Experimental studies and DFT calculations suggested that the reaction likely follows some interesting pathways, which are triggered by a hydride abstraction of pinacolborane with tropylium ion. This is followed by a series of in situ counterion-activated substituent exchanges to generate boron intermediates that promote the hydroboration reaction.

\section{Acknowledgements}

This work was funded by the Australian Research Council (grant FT180100260 and DP200100063 to TVN).
Keywords: tropylium $\cdot$ hydroboration $\cdot$ boron $\cdot$ carbocation $\bullet$ catalysis

[1] a) J. Carreras, A. Caballero, P. J. Pérez, Chem. Asian J. 2019, 14, 329343; b) C. C. Chong, R. Kinjo, ACS Catal. 2015, 5, 3238-3259.

[2] a) H. C. Brown, B. C. S. Rao, J. Am. Chem. Soc. 1956, 78, 5694-5695; b) H. C. Brown, G. Zweifel, J. Am. Chem. Soc. 1961, 83, 486-487; c) H. C. Brown, K. Murray, J. Am. Chem. Soc. 1959, 81, 4108-4109.

[3] a) I. Beletskaya, A. Pelter, Tetrahedron 1997, 53, 4957-5026; b) G. J. Irvine, M. J. G. Lesley, T. B. Marder, N. C. Norman, C. R. Rice, E. G. Robins, W. R. Roper, G. R. Whittell, L. J. Wright, Chem. Rev. 1998, 98 2685-2722; c) I. Beletskaya, C. Moberg, Chem. Rev. 2006, 106, 23202354.

[4] a) S. Pereira, M. Srebnik, Organometallics 1995, 14, 3127-3128; b) C. Gunanathan, M. Hölscher, F. Pan, W. Leitner, J. Am. Chem. Soc. 2012 134, 14349-14352; c) S Mandal, P. K Verma, K Geetharani, Chem. Commun. 2018, 54, 13690-13693; d) K. Yamamoto, Y. Mohara, Y. Mutoh, S. Saito, J. Am. Chem. Soc. 2019, 141, 17042-17047; e) C. J. Lata, C. M. Crudden, J. Am. Chem. Soc. 2010, 132, 131-137; f) R. Mamidala, V. K. Pandey, A. Rit, Chem. Commun. 2019, 55, 989-992; g) Y. Wang, R. Guan, P. Sivaguru, X. Cong, X. Bi, Org. Lett. 2019, 21, 4035-4038.

[5] a) C. K. Blasius, V. Vasilenko, R. Matveeva, H. Wadepohl, L. H. Gade, Angew. Chem. Int. Ed. 2020, 59, 23010-23014; b) K. Semba, T. Fujihara, J. Terao, Y. Tsuji, Chem. Eur. J. 2012, 18, 4179-4184; c) J. V. Obligacion, P. J. Chirik, Org. Lett. 2013, 15, 2680-2683; d) J. V. Obligacion, J. M. Neely, A. N. Yazdani, I. Pappas, P. J. Chirik, J. Am. Chem. Soc. 2015, 137, 5855-5858; e) W. J. Jang, W. L. Lee, J. H. Moon, J. Y. Lee, J. Yun, Org. Lett. 2016, 18, 1390-1393; f) H. Yoshida, ACS Catal. 2016, 6, 1799$1811 ;$ g) N. Gorgas, L. G. Alves, B. Stöger, A. M. Martins, L. F. Veiros, K. Kirchner, J. Am. Chem. Soc. 2017, 139, 8130-8133; h) Q. Huang, M.-Y. 
Hu, S.-F. Zhu, Org. Lett. 2019, 21, 7883-7887; i) L. E. Longobardi, A Fürstner, Chem Eur J. 2019, 25, 10063-10068; j) S. Mandal, S. Mandal, K. Geetharani, Chem. Asian J. 2019, 14, 4553-4556; k) H. L. Sang, C Wu, G. G. D. Phua, S. Ge, ACS Catal. 2019, 9, 10109-10114; I) G. Zhang S. Li, J. Wu, H. Zeng, Z. Mo, K. Davis, S. Zheng, Org. Chem. Front. 2019 , 6, 3228-3233.

[6] a) A. Bismuto, S. P. Thomas, M. J. Cowley, Angew. Chem. Int. Ed. 2016 55, 15356-15359; b) Z. Yang, M. Zhong, X. Ma, K. Nijesh, S. De, P Parameswaran, H. W. Roesky, J. Am. Chem. Soc. 2016, 138, 25482551; c) Y. Wu, C. Shan, J. Ying, J. Su, J. Zhu, L. L. Liu, Y. Zhao, Green Chem 2017, 19, 4169-4175; d) A. Bismuto, M. J. Cowley, S. P. Thomas, ACS Catal. 2018, 8, 2001-2005; e) M. K. Bisai, S. Yadav, T. Das, K Vanka, S. S. Sen, Chem. Commun. 2019, 55, 11711-11714; f) A. K Jaladi, H. Kim, J. H. Lee, W. K. Shin, H. Hwang, D. K. An, New J. Chem. 2019, 43, 16524-16529; g) D. Yan, X. Wu, J. Xiao, Z. Zhu, X. Xu, X. Bao, Y. Yao, Q. Shen, M. Xue, Org. Chem. Front. 2019, 6, 648-653; h) X. Cao, J. Li, A. Zhu, F. Su, W. Yao, F. Xue, M. Ma, Org. Chem. Front. 2020; i) A. D. C. p. Sadow, 2020; j) N. Sarkar, S. Bera, S. Nembenna, J. Org Chem. 2020, 85, 4999-5009; k) V. Vasilenko, C. K. Blasius, H. Wadepohl L. H. Gade, Chem. Commun 2020, 56, 1203-1206; I) K. Revunova, G. I. Nikonov, Dalton Trans. 2015, 44, 840-866; m) M. S. Hill, D. J. Liptrot, C. Weetman, Chem. Soc. Rev. 2016, 45, 972-988.

[7] a) K. Shirakawa, A. Arase, M. Hoshi, Synthesis 2004, 2004, 1814-1820 b) R. P. Rucker, A. M. Whittaker, H. Dang, G. Lalic, J. Am. Chem. Soc. 2012, 134, 6571-6574; c) M. Fleige, J. Möbus, T. vom Stein, F. Glorius, D. W. Stephan, Chem. Commun. 2016, 52, 10830-10833; d) J. S. McGough, S. M. Butler, I. A. Cade, M. J. Ingleson, Chem. Sci. 2016, 7 3384-3389; e) Q. Yin, S. Kemper, H. F. T. Klare, M. Oestreich, Chem Eur. J. 2016, 22, 13840-13844; f) J. R. Lawson, R. L. Melen, Inorg. Chem. 2017, 56, 8627-8643; g) J. R. Lawson, L. C. Wilkins, R. L. Melen, Chem. Eur. J. 2017, 23, 10997-11000; h) N. W. J. Ang, C. S. Buettner, S Docherty, A. Bismuto, J. R. Carney, J. H. Docherty, M. J. Cowley, S. P Thomas, Synthesis 2018, 50, 803-808; i) J. L. Carden, L. J. Gierlichs, D. F. Wass, D. L. Browne, R. L. Melen, Chem. Commun. 2019, 55, 318-321 j) E. Nieto-Sepulveda, A. D. Bage, L. A. Evans, T. A. Hunt, A. G. Leach, S. P. Thomas, G. C. Lloyd-Jones, J. Am. Chem. Soc. 2019, 141, 18600 18611 ; k) E. A. Patrick, W. E. Piers, Chem. Commun. 2020, 56, 841-853.

[8] a) H. E. Ho, N. Asao, Y Yamamoto, T Jin, Org Lett 2014, 16, 4670 4673; b) C. B. Hoyt, M. L. Sarazen, C. W. Jones, J. Catal. 2019, 369, 493-500.

[9] C. E. Garrett, G. C. Fu, J. Org. Chem. 1996, 61, 3224-3225

[10] a) T. V. Nguyen, A Bekensir, Org Lett. 2014, 16, 1720-1723; b) T. V. Nguyen, M. Hall, Tetrahedron Lett. 2014, 55, 6895-6898; c) T. V. Nguyen, D. J. M. Lyons, Chem. Commun. 2015, 51, 3131-3134; d) D. J. M. Lyons, R. D. Crocker, M. Blümel, T. V. Nguyen, Angew. Chem. Int. Ed. 2017, 56 1466-1484; e) D. Lyons, R. Crocker, V. Nguyen Thanh, Chem. Eur. J. 2018, $24,10959-10965$; f) M. A. Hussein, U. P. N. Tran, V. T. Huynh, J. Ho, M. Bhadbhade, H. Mayr, T. V. Nguyen, Angew. Chem. Int. Ed. 2020 59, 1455-1459; g) D. J. M. Lyons, C. Empel, D. P. Pace, A. H. Dinh, B. K. Mai, R. M. Koenigs, T. V. Nguyen, ACS Catal. 2020, 12596-12606.

[11] a) D. J. M. Lyons, R. D. Crocker, D. Enders, T. V. Nguyen, Green Chem. 2017, 3993-3996; b) M. A. Hussein, V. T. Huynh, R. Hommelsheim, R. M. Koenigs, T. V. Nguyen, Chem. Commun. 2018, 54, 12970-12973; c) G. Oss, J. Ho, V. Nguyen Thanh, Eur. J. Org. Chem. 2018, 3974-3981 d) U. P. N. Tran, G. Oss, D. P. Pace, J. Ho, T. V. Nguyen, Chem. Sci. 2018, 9, 5145-5151; e) K. Omoregbee, K. N. H. Luc, A. H. Dinh, T. V. Nguyen, J. Flow Chem. 2020, 10, 161-166; f) C. Empel, T. V. Nguyen, R. M. Koenigs, Org. Lett. 2021, 23, 548-553.

[12] a) G. Oss, S. D. de Vos, K. N. H. Luc, J. B. Harper, T. V. Nguyen, J. Org Chem. 2018, 83, 1000-1010; b) J. M. Allen, T. H. Lambert, J. Am. Chem. Soc. 2011, 133, 1260-1262; c) M. Horn, L. H. Schappele, G. LangWittkowski, H. Mayr, A. R. Ofial, Chem. Eur. J. 2013, 19, 249-263.

[13] See the experimental Supporting Information for further details.

14] M. Rachwalak, J. Gołębiewska, T. Jakubowski, J. Stawinski, Synthesis

[15] L. Bering, A. P. Antonchick, Tetrahedron 2019, 75, 1131-1143.

[16] a) A. D. Bage, T. A. Hunt, S. P. Thomas, Org. Lett. 2020, 22, 4107-4112 b) A. D. Bage, K. Nicholson, T. A. Hunt, T. Langer, S. P. Thomas, ACS Catal. 2020, 10, 13479-13486.

[17] a) M. A Dureen, A Lough, T. M. Gilbert D. W Stephan, Chem Commun. 2008, 4303-4305; b) A. Bismuto, G. S. Nichol, F. Duarte, M. J. Cowley, S. P. Thomas, Angew. Chem. Int. Ed. 2020, 59, 12731-12735

[18] Z. Li, V. Gandon, C. Bour, Chem. Commun. 2020, 56, 6507-6510.

19] DFT calculations were performed at the PCM/MN15/6$311+G(2 d, 2 p) / / P C M / M 06-2 X / 6-31+G(d, p)$ level of theory. See the computational Supporting Information for more details.

[20] Similar outcomes were observed for HBpin and 1a mixture at $70^{\circ} \mathrm{C}$ after a few minutes of reaction time.

[21] a) A J Cresswell, S. G Davies, A L A Figuccia, A M. Fletcher, D. Heijnen, J. A. Lee, M. J. Morris, A. M. R. Kennett, P. M. Roberts, J. E. Thomson, Tetrahedron Lett. 2015, 56, 3373-3377; b) L. Kuehn, M. Stang S. Würtemberger-Pietsch, A. Friedrich, H. Schneider, U. Radius, T. B. Marder, Faraday Discussions 2019, 220, 350-363.

[22] R. Agahi, A. J. Challinor, N. B. Carter, S. P. Thomas, Org. Lett. 2019, 21 993-997.

[23] T. C. Farrar, T. D. Coyle, J. Chem. Phys. 1964, 41, 2612-2613.
[24] a) H. C. Brown, J. A. Sikorski, Organometallics 1982, 1, 28-37; b) M. E. Schwartz, L. C. Allen, J. Am Chem. Soc. 1970, 92, 1466-1471.

[25] J. W. Clary, T. J. Rettenmaier, R. Snelling, W. Bryks, J. Banwell, W. T. Wipke, B. Singaram, J. Org. Chem. 2011, 76, 9602-9610.

[26] It should be noted that in the case of $\mathrm{Trop} \mathrm{BF}_{4} \mathbf{1 a}$ it is also possible to form $\mathrm{BH}_{2} \mathrm{~F}$ via a similar pathway, i.e. with production $\mathrm{B}_{2}$ pin $_{3}$. However, the activation barrier to form $\mathrm{B}_{2} \mathrm{pin}_{3}$ is $29.5 \mathrm{kcal} / \mathrm{mol}$ relative to $\mathrm{FBpin}$ $\left(+\mathrm{BF}_{3}\right)$. This barrier is $1.9 \mathrm{kcal} / \mathrm{mol}$ higher in energy than the formation of $\mathrm{BH}_{2} \mathrm{~F}$ as depicted in Figure 3a (see Figure S9 in the computational ESI for detail).

[27] M. Magre, E. Paffenholz, B. Maity, L. Cavallo, M. Rueping, J. Am. Chem. Soc. 2020, 142, 14286-14294. 
